\title{
Screening with the Korean Version of the Mood Disorder Questionnaire for Bipolar Disorders in Adolescents: Korean Validity and Reliability Study
}

\author{
Se-Hoon Shim ${ }^{1}$, Jonghun Lee ${ }^{2}$, Jye-Heon Song ${ }^{3}$, Beomwoo Nam ${ }^{4}$, Bo-Hyun Yoon ${ }^{3}$, Ha-young Jin ${ }^{5}$, \\ Hyung-Mo Sung ${ }^{6}$, Jong-Hyun Jeong ${ }^{7}$, Sae-Heon Jang ${ }^{8}$, Duk-In Jon ${ }^{9}$, Young Sup Woo ${ }^{7}$, Won-Myong Bahk ${ }^{7}$ \\ ${ }^{1}$ Department of Psychiatry, Soonchunhyang University Cheonan Hospital, Soonchunhyang University College of Medicine, Cheonan, ${ }^{2}$ Department \\ of Psychiatry, Catholic University of Daegu College of Medicine, Daegu, ${ }^{3}$ Department of Psychiatry, Naju National Hospital, Naju, ${ }^{4}$ Department \\ of Psychiatry, Konkuk University School of Medicine, Chungju, ${ }^{5}$ Department of Psychology, Chungbuk National University, Cheongju, \\ ${ }^{6}$ Department of Psychiatry, CHA University, Gumi, ${ }^{7}$ Department of Psychiatry, College of Medicine, The Catholic University of Korea, Seoul, \\ ${ }^{8}$ Department of Psychiatry, Bongseng Memorial Hospital, Busan, ${ }^{9}$ Department of Psychiatry, Hallym University Sacred Heart Hospital, Hallym \\ University College of Medicine, Anyang, Korea
}

\begin{abstract}
Objective: This study aimed to evaluate the validity and reliability of a Korean version of the Mood Disorder Questionnaire-Adolescent version (K-MDQ-A) as a screening instrument for bipolar disorders in adolescents.

Methods: One hundred two adolescents with bipolar disorders and their parents were recruited from November 2014 to November 2016 at 7 training hospitals. One hundred six controls were recruited from each middle school in two cities of South Korea. The parent version of the original MDQ-A was translated into Korean. The parents of all participants completed the K-MDQ-A. The diagnoses of bipolar disorders were determined based on the Korean version of K-SADS-PL. The test-retest reliability with a 10-month interval was investigated in 33 bipolar adolescents.

Results: K-MDQ-A yielded a sensitivity of 0.90 and a specificity of 0.92 when using a cut-off score of endorsement of 5 items, indicating that symptoms occurred in the same time period and caused moderate or serious problems. The internal consistency of the K-MDQ-A was good. The correlations between each item and the total score ranged from 0.40 to 0.76 and were all statistically significant. Factor analysis revealed 3 factors that explained $61.25 \%$ of the total variance. The mean total score was significantly higher in bipolar adolescents (7.29) than in controls (1.32). The Pearson correlation coefficient for the total test-retest score was $0.59(p<0.001)$.

Conclusion: The K-MDQ-A completed by parents showed the excellent validity and reliability and may be a useful screening tool for adolescents with bipolar disorders attending in- and outpatient psychiatric clinics.
\end{abstract}

KEY WORDS: Adolescent; Bipolar disorder; Mood Disorder Questionnaire; Reliability and validity; Screening.

\section{INTRODUCTION}

In the Systematic Treatment Enhancement Program for

\footnotetext{
Received: August 4, 2017 / Revised: August 25, 2017

Accepted: August 26, 2017

Address for correspondence: Hyung-Mo Sung, MD, PhD Department of Psychiatry, CHA University, 12 Sinsi-ro 10-gil, Gumi 39295, Korea

Tel: +82-54-450-9678, Fax: +82-54-452-5098

E-mail: huyngmo@hanmail.net

ORCID: https://orcid.org/0000-0002-2396-3358

Won-Myong Bahk, MD, PhD

Department of Psychiatry, Yeouido St. Mary's Hospital, College of

Medicine, The Catholic University of Korea, 10 63-ro,

Yeongdeungpo-gu, Seoul 07345, Korea

Tel: +82-2-3779-1250, Fax: +82-2-780-6577

E-mail:wmbahk@catholic.ac.kr

ORCID: https://orcid.org/0000-0002-0156-2510
}

Bipolar Disorder, $27.7 \%$ of the first consecutive 1,000 adult bipolar patients developed bipolar disorder at a very young age (age, <13 years), and 37.6\% developed bipolar disorder at a young age (age, 13-18 years). Early onset was associated with greater likelihood of comorbid anxiety disorders and substance abuse, more recurrences, shorter euthymic periods, and greater rates of suicide attempts and violence. ${ }^{1)}$ Thus, bipolar disorders have their onset at an early age, with a mean onset between the ages of 15 and 19 years, as reported in reviews on the initial prodrome and recognition of bipolar disorders ${ }^{2,3)}$ and they follow a serious course of illness. However, there is skepticism about the prevalence, validity and stability of bipolar disorder in children and adolescents outside the

(c) This is an Open-Access article distributed under the terms of the Creative Commons Attribution Non-Commercial License (http://creativecommons.org/licenses/by-nc/4.0) which permits unrestricted non-commercial use, distribution, and reproduction in any medium, provided the original work is properly cited. 
United States ${ }^{4)}$ bipolar disorder is rarely diagnosed, and there is no epidemiological report in children and adolescents in South Korea.

Inaccurate or missed diagnosis of bipolar disorder in childhood may lead to poor prognosis. Many children and adolescents with bipolar disorders are not treated appropriately. ${ }^{5,6)}$ When bipolar disorder has in essence occurred, the appropriate intervention in the early stage should prevent progress of the disease. Interventions in the earliest phases give an opportunity to prevent the disease from progressing and resulting in undesirable sequelae in high risk youths with such as family history. ${ }^{7)}$ Therefore, early identification and intervention for children and adolescents with bipolar disorders need particular consideration. ${ }^{8)}$

To improve the diagnosis of bipolar disorders among children and adolescents in different practices, validity and reliability of questionnaires must be assessed using multiple informant strategies. ${ }^{9)}$ Therefore, a screening tool for bipolar disorders including I, II, not otherwise specified (NOS), and cyclothymia in an adolescent population, the Mood Disorder Questionnaire-Adolescent version (MDQ-A), has been developed. In accordance with the original MDQ-A validation study, the parent report was superior to the adolescent self-report and attributional report in terms of how the adolescent believed teachers or friends would describe one's symptoms, for sensitivity and specificity in an outpatient psychiatric population. ${ }^{10}$

Moreover, although screening tools for bipolar disorder in adults are translated and used in Korea, ${ }^{11,12)}$ the tools for children and adolescents are not yet available in Korea. Hence, this study aimed to test the Korean version of the parent report of the MDQ-A (K-MDQ-A) in an adolescent in- and outpatient psychiatric population. We examined the test-retest reliability of the K-MDQ-A, as well as the validity of both instruments with respect to the reference diagnosis based on the Korean version of the Schedule for Affective Disorders and Schizophrenia for School Age Children-Present and Lifetime version (K-SADS-PL). ${ }^{13)}$

\section{METHODS}

\section{Participants and Procedure}

This study was conducted at the outpatient and inpatient facilities of seven training hospitals (five university hospitals and two mental hospitals) that treat adolescents living in seven cities of South Korea. One hundred two adolescents with bipolar disorder were recruited from November 2014 to November 2016 from all sites. The diagnosis of bipolar disorder was determined based on the Korean version of K-SADS-PL, a semi-structured diagnostic interview based on the Diagnostic and Statistical Manual of Mental Disorders 4th edition (DSM-IV) criteria, by each author who was a life member of the Korean Society for Mood Disorders and an expert in the field of mood disorders or child and adolescent psychiatry. The control group consisted of students who were recruited from each middle school in two cities of Korea. All 106 controls were academically well-adjusted, physically healthy and showed no signs of psychiatric or developmental problems. All participants met the age criteria of age between 12 and 17 years. The parents of the participants completed the K-MDQ-A. The test-retest reliability was examined, at an interval of about 10 months between assessments, among 33 adolescents by some authors (H.M.S, J.H.L., and S.H.S).

Written informed consent was obtained from all participants and their parents. The protocol for the research project has been approved by a suitably constituted Ethics Committee of an institution, and it is conformed to the provisions of the Declaration of Helsinki. The institutional review board of the participating hospitals approved this study protocol (Soonchunhyang University Cheonan Hospital, Catholic University of Daegu, Naju National Hospital, Konkuk University Chungju Hospital, CHA University Gumi Medical Center, The Catholic University of Korea, Bongseng Memorial Hospital).

The clinician who administered the K-SADS-PL diagnostic interview was blinded to the results of the K-MDQ-A. K-MDQ-A items were summed, yielding a score for each adolescent ranging from 0 to 13 . Each possible scoring threshold, in combination with co-occurrence of symptoms and behaviors and with moderate to serious problems caused by symptoms, was assessed based on the results of the K-SADS-PL diagnostic interview to yield sensitivity and specificity.

\section{Instruments}

With the assent of the author of the original MDQ-A, the MDQ-A was translated into Korean by a child and adolescent psychiatrist (S.H.S.). This preliminary translated questionnaire was applied to several persons who 
had no psychiatric illness. The K-MDQ-A was back-translated into English by a bilingual psychiatrist who did not know the original MDQ-A. All authors reviewed and reconfirmed the results before developing the final version.

The K-MDQ-A is composed of 3 parts. Part 1 is the K-MDQ-A screens for a lifetime history of manic or hypomanic symptoms using 13 yes/no items. Part 2 enquires whether co-occurrence of symptoms and behaviors has been experienced, and part 3 assesses from "no" to "serious" problems due to symptoms on a 4-point scale. In the original MDQ-A, informants are considered positive for bipolar disorder using a cut-off score of 5 items for the symptoms in part 1 , answer "yes" in part 2, and indicate that the symptoms result in "moderate" to "severe" problems in part 3.

\section{Data Analysis}

The internal consistency of the K-MDQ-A was evaluated with Cronbach's $\alpha$. For construct validity, we conducted factor analysis with extraction of principal components using varimax rotation. Correlation analyses were conducted between each questionnaire item and the total K-MDQ-A score. We compared the frequency of each questionnaire item and the total K-MDQ-A score between 2 groups with the $t$ test. The optimal cut score was determined by computing the sensitivity and specificity for various cutoff scores. Receiver operating characteristic (ROC) curves were calculated for the K-MDQ-A, together

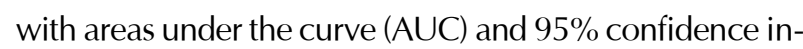
terval $(\mathrm{Cl})$. For the reliability of test-retest, we estimated the Pearson correlation coefficient. Analysis of variance (ANOVA) was used to determine the influence of change in the clinical phase on the total K-MDQ-A score in 33 subjects assessed for test-retest reliability. Statistical significance was set at 0.05 (2-tailed exact tests). Data analysis was performed using SPSS version 17.0 (SPSS Inc., Chicago, IL, USA).

\section{RESULTS}

The demographic and clinical characteristics of adolescents with bipolar disorders and controls are presented in Table 1. There was no significant difference in gender $\left(\chi^{2}=4.46, p<0.001\right)$ and age $(\mathrm{t}=4.42, p<0.001)$ between the two groups. The mean duration of illness in adolescents with bipolar disorders was 14.34 months and it ranged from less than 1 month to 96 months. The diagnosis of bipolar disorder in adolescents was as follows: bipolar I disorder $(n=43,42.2 \%)$, bipolar II disorder $(n=5$, $4.9 \%$ ), and bipolar disorder NOS ( $n=54,52.9 \%$ ). Comorbid disorders (number [\%]) in the bipolar group were as follows: attention-deficit/hyperactivity disorder (31 [31.6\%]), oppositional defiant disorder/conduct disorder $(7[7.1 \%])$, anxiety disorder $(29[29.6 \%])$, tic disorder $(10[10.2 \%])$, and posttraumatic stress disorder (1 $[1.2 \%])$.

The mean hospitalization numbers for adolescents with bipolar disorders bipolar disorder was about 0.6 (range, $0-2)$, and $45(44.1 \%)$ adolescents had never been admitted before this study.

The K-MDQ-A had good internal consistency (Cronbach's $\alpha=0.881$ ). Correlation coefficients between each questionnaire and the total K-MDQ-A score ranged from 0.40 ("consumed more alcohol or drugs") to 0.76 ("could not slow down his/her mind or thoughts raced through his/her head") and all of them were statistically significant ( $p<$ 0.001).

Three factors (composed of 6, 5, and 2 questionnaires) with eigenvalues greater than 1 were extracted from factor analysis, and they explained $61.25 \%$ of the total variance. Factor 1 was the group of 6 activation items, accounting for $24.15 \%$ of the variance. The remaining 2 factors totally contributed to $37.10 \%$ of the variance (Table 2).

The frequency of endorsement of each K-MDQ-A questionnaire was from $22.5 \%$ to $73.5 \%$ in the bipolar group and from $0.9 \%$ to $22.6 \%$ in the control group (Fig. 1). The

Table 1. Demographic characteristics of all participants

\begin{tabular}{lcccc}
\hline \multicolumn{1}{c}{ Characteristic } & Control $(\mathrm{n}=106)$ & Bipolar disorder $(\mathrm{n}=102)$ & Statistical parameter & $p$ value \\
\hline Sex, male:female & $61: 45$ & $54: 48$ & $\chi^{2}=0.446$ & $\mathrm{NS}$ \\
Age (yr) & $14.35 \pm 1.63$ & $15.38 \pm 1.74$ & $\mathrm{t}=4.42$ & $\mathrm{NS}$ \\
Duration of illness (mo) & & $14.34 \pm 15.61$ & & \\
\hline
\end{tabular}

Values are presented as number only or mean \pm standard deviation.

NS, non-significance. 
highest questionnaire endorsement was given to "felt too good or excited" in adolescents with bipolar disorders and to "felt he/she could do anything" in the control group. In the control group, the frequencies of all items were less than $25 \%$. The endorsements of all questionnaires were significantly higher in the patient group than in the control group. The item "felt too good or excited" differed the most between the two groups $(\mathrm{t}=11.34, p<0.001)$. The score of each item $(p<0.001)$ as well as the total K-MDQ-A score (7.29 \pm 2.86 vs. $1.32 \pm 1.81 ; \mathrm{t}=17.99, p<$ 0.001 ) were significantly higher in adolescents with bipolar disorders than in controls.

Sensitivity and specificity analyses were conducted for the K-MDQ-A using K-SADS-PL bipolar diagnosis as the standard. Figure 2 presents the sensitivity and specificity with various cut off scores of the total K-MDQ-A score. In

Table 2. Factor loadings of the K-MDQ-A from principal-components analysis with varimax rotation in all participants

\begin{tabular}{|c|c|c|c|}
\hline K-MDQ-A item & Factor 1 & Factor 2 & Factor 3 \\
\hline 2 so irritable & 0.776 & & \\
\hline 6 so easily distracted by things & 0.769 & & \\
\hline 11 many things that were foolish or risky & 0.693 & & \\
\hline 5 couldn't slow his/her mind down & 0.650 & & \\
\hline 12 spent too much money & 0.459 & & \\
\hline 10 more interested in sex & 0.478 & & \\
\hline 8 much more active & & 0.794 & \\
\hline 7 much more energy & & 0.788 & \\
\hline 3 could do anything & & 0.764 & \\
\hline 1 too good or excited & & 0.590 & \\
\hline 4 needed much less sleep & & 0.422 & \\
\hline 13 used more alcohol or drugs & & & 0.850 \\
\hline 9 boyfriends or girlfriends at the same time & & & 0.804 \\
\hline Eigenvalue & 3.14 & 2.85 & 1.97 \\
\hline Total variance explained (\%) & 24.15 & 21.92 & 15.18 \\
\hline
\end{tabular}

K-MDQ-A, Korean version of the Mood Disorder QuestionnaireAdolescent version. the K-MDQ-A, the parent-reported data yielded a sensitivity of $90 \%$ and a specificity of $92 \%$ when using a cut-off score of endorsement of 5 items (part 1), indicating that symptoms occurred in the same time period (part 2 [symptoms clustering]) and caused moderate or serious problems (part 3 [functional impairments]).

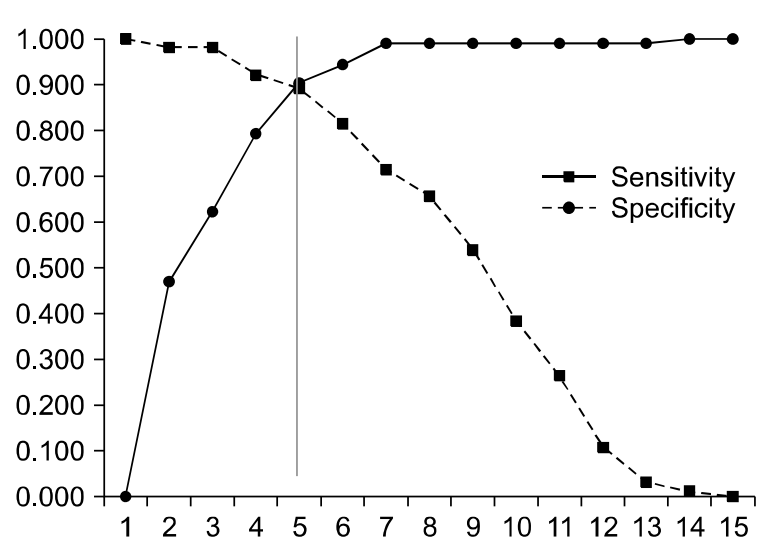

Fig. 2. Operating characteristics of Korean Mood Disorder Questionnaire-Adolescent version.

Table 3. Responses of adolescents with bipolar I disorder, bipolar II disorder or bipolar disorder not otherwise specified

\begin{tabular}{lrrrrr}
\hline & \multicolumn{5}{c}{ Bipolar disorder type } \\
\cline { 2 - 6 } & I & II & NOS & $\chi^{2}$ & $p$ value \\
\hline Total score of 15 items & 5 & 1 & 4 & & NS \\
$\quad<5$ & 38 & 4 & 50 & & \\
$\quad \geq 5$ & & & & 0.981 & NS \\
$\quad \begin{array}{l}\text { Symptoms clustering } \\
\quad \text { No }\end{array}$ & 4 & 0 & 9 & & \\
$\quad$ Yes & 39 & 5 & 45 & & \\
$\begin{array}{l}\text { Seriousness of problems } \\
\quad \text { No" or "Minor" }\end{array}$ & 8 & 1 & 14 & & \\
$\quad$ "Moderate" or "Serious" & 35 & 4 & 40 & & \\
\hline
\end{tabular}

NOS, not otherwise specified; NS, non-significance.

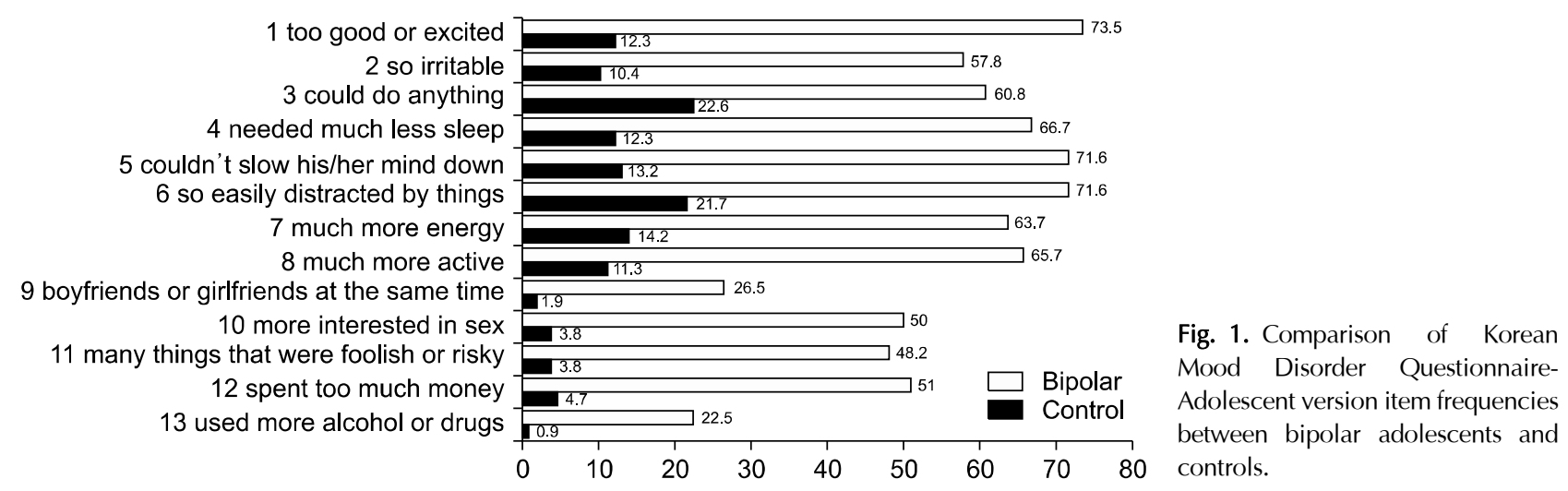


Table 4. Changes in the clinical phase and the total K-MDQ-A score of 33 bipolar patients assessed for test-retest reliability

\begin{tabular}{|c|c|c|c|c|c|}
\hline \multirow{2}{*}{$\begin{array}{l}\text { Clinical phase } \\
\text { (time } 1 \text { to time } 2 \text { ) }\end{array}$} & \multirow{2}{*}{ Subject (n) } & \multicolumn{2}{|c|}{ Total K-MDQ score } & \multirow{2}{*}{$\mathrm{t}$} & \multirow{2}{*}{$p$ value } \\
\hline & & Time 1 & Time 2 & & \\
\hline Not changed & 21 & $7.81 \pm 1.69$ & $7.76 \pm 1.70$ & 1.00 & 0.329 \\
\hline Euthymic-euthymic & 11 & $8.36 \pm 1.63$ & $8.36 \pm 1.63$ & & \\
\hline Manic-manic & 3 & $8.33 \pm 1.53$ & $8.33 \pm 1.53$ & & \\
\hline Hypomanic-hypomanic & 2 & 8 & $7.50 \pm 0.71$ & 1.00 & 0.500 \\
\hline Depressed-depressed & 5 & $6.20 \pm 1.48$ & $6.20 \pm 1.48$ & & \\
\hline Changed & 12 & $8.92 \pm 2.50$ & $9.00 \pm 2.49$ & -1.000 & 0.339 \\
\hline \multicolumn{6}{|l|}{ Upward } \\
\hline Depressed-euthymic & 3 & $8.33 \pm 2.89$ & $8.33 \pm 2.89$ & & \\
\hline Downward & 9 & $9.11 \pm 2.52$ & $9.22 \pm 2.49$ & -1.00 & 0.347 \\
\hline Manic-euthymic & 6 & $8.83 \pm 2.99$ & $9.00 \pm 2.97$ & -1.00 & 0.363 \\
\hline Manic-depressed & 1 & 10 & 10 & & \\
\hline Hypomanic-euthymic & 2 & $9.50 \pm 2.12$ & $9.50 \pm 2.12$ & & \\
\hline Total & 33 & $8.21 \pm 2.06$ & $8.21 \pm 2.07$ & 0.00 & 1.00 \\
\hline
\end{tabular}

Values are presented as number only or mean \pm standard deviation.

K-MDQ-A, Korean version of the Mood Disorder Questionnaire- Adolescent version.

There was no statistical difference in parts 1, 2 and 3 among bipolar I disorder, bipolar II disorder, and bipolar disorder NOS (Table 3).

ROC plots for the K-MDQ-A are depicted in Figure 2. Corresponding AUC values were $0.96(95 \% \mathrm{Cl}, 0.94$ 0.99) at the usual threshold of the 5 endorsed symptoms for the K-MDQ-A.

For the reliability of test-retest, we estimated the Pearson correlation coefficient for the total K-MDQ-A scores with a 10-month interval. The Pearson correlation coefficient for the total K-MDQ-A score was 0.59 ( $p<$ 0.001). The K-MDQ-A showed excellent test-retest reliability over 10 months. As indicated in Table 4, the most common change pattern in the clinical phase was euthymic-euthymic (33.3\%). ANOVA represented that there were not any significant differences between the two check points of the total K-MDQ-A score in the unchanged $(n=21)$, changed upward $(n=3)$ and changed downward groups $(n=9)(F=0.253$, degree of freedom $=1$, $p=0.620$ ).

\section{DISCUSSION}

In the present study, we translated the MDQ- $\mathrm{A}^{10)}$ into Korean and assessed the validity and reliability of the K-MDQ-A.

The original MDQ-A showed good validity and reliability when responses were obtained from parents about symptoms in children and adolescents attending out- patient psychiatric clinics. However, self-report by adolescents and report by others who viewed the adolescent's behavior were less useful in screening for bipolar disorders. Therefore, we just used the parent version of the original MDQ-A. The K-MDQ-A as a screen for bipolar disorders in adolescents was excellent (sensitivity of $90 \%$ and specificity of $92 \%$ ) when parents also indicated that symptoms occurred in the same time period and caused moderate or serious problems in addition to endorsement of 5 items. Similar to the present study, using a cut-off score of the endorsement of 5 items in the original MDQ-A, the parent-reported data yielded a good validity such as sensitivity of $72 \%$ and specificity of $81 \%$, which were superior to the values obtained by using self and attribution versions. The using 6 more items in K-MDQ-A as cut score resulted in significant loss of sensitivity in agreement with the original MDQ-A.

Corresponding AUC values of the ROC plots are excellent at the usual threshold of 5 endorsed symptoms for the K-MDQ-A, similar to those in previous studies. For the K-MDQ-A, best discrimination between adolescents with bipolar disorders and controls (best values for sensitivity and specificity) occurred at a cut-off score of 5 positive items with affirmation to parts 2 and 3 .

Compared to validation studies of the original MDQ-A for parents and the French version of the MDQ- $A^{4)}$ for parents, the K-MDQ-A showed better sensitivity (90\% vs. $72 \%$ [original] $/ 88 \%$ [French version]) and specificity (92\% vs. 81\% [original]/63\% [French version]). 
The MDQ, a valid instrument for screening of bipolar spectrum disorders in adults, proposed that participants with seven more symptoms and some symptoms occurring at the same time with "moderate" or "serious" problems should be diagnosed as having bipolar disorders. ${ }^{14)}$ The optimal cut-off of Chinese version of MDQ in an outpatient psychiatric population in Hong Kong was 7 or more manic symptoms occurring at the same time period without the impairment criterion. ${ }^{15)}$ On the other hand, In the $\mathrm{K}-\mathrm{MDQ}{ }^{12)} 7$ more total score without affirmation to parts 2 and 3 was chosen as the best standard in the validation study. The authors proposed the optimal cut-off items that were best standard for each study. The more stringent the criteria, the lower the sensitivity. Compared to validation studies of the all MDQs for adult, the K-MDQ-A displayed better sensitivity.

The factors due to which the K-MDQ-A has better sensitivity and specificity may be associated with participants' characteristics. This study included in- and outpatients attending the training hospitals that treat adolescents and are specialized in mood disorders or child and adolescent psychiatry. Thus, parents had specific education on bipolar disorders. In the original MDQ-A study, there was little information on recruitment; however, it included outpatients and relatively fewer adolescents with bipolar disorders. The French version study included a very low number of adolescents with K-SADS-PL-confirmed bipolar disorders. Although the K-MDQ-A showed excellent validity and reliability, we must carefully consider the followings and use. First, adolescents have relatively non-specific symptoms such as insomnia, physical restlessness, distractibility, racing thoughts, pressured speech, intrusiveness, temper outbursts and irritability. ${ }^{16)}$ Second, screening tools may detect subthreshold manic symptoms in adolescence but infrequently predict bipolar disorder requiring mental health care use. ${ }^{17)}$ Third, adolescent subsyndromal bipolar disorder was associated with poor outcomes including major depressive disorder and anxiety disorders in young adulthood, but was not associated with an increased incidence of bipolar disorder. ${ }^{18)}$ Fourth, bipolar disorder may first manifest in the form of depression. ${ }^{19)}$ Fifth, the symptoms of bipolar disorder overlap and may be confused with symptoms of other common psychiatric disorders in children including attention-deficit/hyperactivity disorder, oppositional defiant disorder and conduct disorder. ${ }^{20)}$
The internal consistency of the K-MDQ-A was good and it was consistent with the original MDQ-A. Although the authors of the original MDQ-A briefly reported about sensitivity, specificity, and cut-off value and they did not provide any detailed information on its validation, we analyzed the data that showed various characteristics of validation of the K-MDQ-A. The correlations between each questionnaire and the total score were significant. Factor analysis showed that 3 factors explained $61.25 \%$ of the total variance. The factor with the highest eigenvalue consisted of "was so irritable that he/she started fights or arguments with people," "was so easily distracted by things," "did many things that were foolish or risky," "could not slow down his/her mind or thoughts raced through his/her head," and "was more interested in sex than usual," which are all activation behavior of manic symptoms. Significant differences in all items and the total K-MDQ-A scores between both groups indicate the powerful discrimination competence of this instrument. These results suggest that the K-MDQ-A should be regarded a valid and reliable screening instrument for Korean adolescents with bipolar disorders.

Because the current mood state of adolescents might affect reports by the parents in the K-MDQ-A, this study assessed test-retest reliability to determine the stability of reports over time. Although the original MDQ-A study was not performed to analyze the reliability of reports over time, the K-MDQ-A exhibited good test-retest reliability over 10 months. The total K-MDQ-A scores were not significantly different according to changes in the clinical phase.

The limitation of this study was that it did not consider screening for bipolar disorders in adolescents within primary care settings and the community. Further study is needed to determine whether the K-MDQ-A would be useful in primary care settings and the community. Another limitation of this study was that there is no report of control group from their parents because they were all well-adjusted academically, physically healthy and showed no signs of psychiatric or developmental problems.

The K-MDQ-A is useful in the early recognition of bipolar disorders in clinical situations. With the K-MDQ-A, the clinician can achieve an early diagnosis, perform appropriate treatment and provide a better prognosis for patients with bipolar disorder. Administration of the K-MDQ-A to parents of adolescents attending a psychi- 
atric in- or out-patient clinic may facilitate the identification of bipolar disorders in these youths.

In conclusion, the present study developed and validated the K-MDQ-A, a Korean translated Mood Disorder Questionnaire-Adolescent version for parents. The K-MDQ-A was administered to 102 adolescents with bipolar disorder attending in- and outpatient clinics of training hospitals and truly diagnosed as having bipolar disorder using the K-SADS-PL and to 106 controls who had no history of psychiatric diagnosis or treatment. The K-MDQ-A showed good internal consistency. Each questionnaire and the total K-MDQ-A score were significantly correlated. The frequency of each questionnaire and the total score were significantly higher in the adolescents with bipolar disorder than in the controls. In accordance with the original MDQ-A, the sensitivity and specificity were excellent when using a cut-off score of 5 for the total K-MDQ-A score with co-occurrence of endorsed symptoms and functional problems caused by symptoms. These results propose that the K-MDQ-A is an adequate screening instrument for Korean adolescents with bipolar disorders.

\section{Acknowledgments}

This study was supported by the Korean College of Neuropsychopharmacology, the Korean Society for Affective Disorders, and Soonchunhyang University.

\section{REFERENCES}

1. Perlis RH, Miyahara S, Marangell LB, Wisniewski SR, Ostacher M, DelBello MP, et al. Long-term implications of early onset in bipolar disorder: data from the first 1000 participants in the systematic treatment enhancement program for bipolar disorder (STEP-BD). Biol Psychiatry 2004;55:875-881.

2. Hauser M, Pfennig A, Ozgürdal S, Heinz A, Bauer M, Juckel G. Early recognition of bipolar disorder. Eur Psychiatry 2007;22: 92-98.

3. Skjelstad DV, Malt UF, Holte A. Symptoms and signs of the initial prodrome of bipolar disorder: a systematic review. J Affect Disord 2010;126:1-13.

4. Miguez M, Weber B, Debbané M, Balanzin D, Gex-Fabry M, Raiola F, et al. Screening for bipolar disorder in adolescents with the Mood Disorder Questionnaire-Adolescent version (MDQ-A) and the Child Bipolar Questionnaire (CBQ). Early Interv Psychiatry 2013;7:270-277.

5. Evans-Lacko SE, dosReis S, Kastelic E, Riley AW. Mental health service use before and after diagnosis of early-onset bipolar disorder. J Behav Health Serv Res 2011;38:398-413.
6. Evans-Lacko SE, Dosreis S, Kastelic EA, Paula CS, Steinwachs DM. Evaluation of guideline-concordant care for bipolar disorder among privately insured youth. Prim Care Companion J Clin Psychiatry 2010;12:PCC.09m00837.

7. Muneer A. Staging models in bipolar disorder: a systematic review of the literature. Clin Psychopharmacol Neurosci 2016; 14:117-130

8. Scott J. Bipolar disorder: from early identification to personalized treatment. Early Interv Psychiatry 2011;5:89-90.

9. Moreno C, Laje G, Blanco C, Jiang H, Schmidt AB, Olfson M. National trends in the outpatient diagnosis and treatment of bipolar disorder in youth. Arch Gen Psychiatry 2007;64: 1032-1039.

10. Wagner KD, Hirschfeld RM, Emslie GJ, Findling RL, Gracious BL, Reed ML. Validation of the Mood Disorder Questionnaire for bipolar disorders in adolescents. J Clin Psychiatry 2006; 67:827-830.

11. Yoon BH, Angst J, Bahk WM, Wang HR, Bae SO, Kim MD, et al. Psychometric properties of the Hypomania Checklist-32 in Korean patients with mood disorders. Clin Psychopharmacol Neurosci 2017; 15:352-360.

12. Jon DI, Hong $\mathrm{N}$, Yoon $\mathrm{BH}$, Jung $\mathrm{HY}, \mathrm{Ha} \mathrm{K}$, Shin $\mathrm{YC}$, et al. Validity and reliability of the Korean version of the Mood Disorder Questionnaire. Compr Psychiatry 2009;50:286291.

13. Kaufman J, Birmaher B, Brent D, Rao U, Flynn C, Moreci P, et al. Schedule for Affective Disorders and Schizophrenia for School-Age Children-Present and Lifetime Version (K-SADS-PL): initial reliability and validity data. I Am Acad Child AdolesC Psychiatry 1997;36:980-988.

14. Hirschfeld RM, Williams JB, Spitzer RL, Calabrese JR, Flynn L, Keck PE Jr, et al. Development and validation of a screening instrument for bipolar spectrum disorder: the Mood Disorder Questionnaire. Am J Psychiatry 2000;157:1873-1875.

15. Chung KF, Tso KC, Cheung E, Wong M. Validation of the Chinese version of the Mood Disorder Questionnaire in a psychiatric population in Hong Kong. Psychiatry Clin Neurosci 2008;62:464-471.

16. Brotman MA, Schmajuk M, Rich BA, Dickstein DP, Guyer AE, Costello EJ, et al. Prevalence, clinical correlates, and longitudinal course of severe mood dysregulation in children. Biol Psychiatry 2006;60:991-997.

17. Tijssen MJ, van Os J, Wittchen HU, Lieb R, Beesdo K, Mengelers R, et al. Evidence that bipolar disorder is the poor outcome fraction of a common developmental phenotype: an 8-year cohort study in young people. Psychol Med 2010;40: 289-299.

18. Lewinsohn PM, Klein DN, Seeley JR. Bipolar disorder during adolescence and young adulthood in a community sample. Bipolar Disord 2000;2:281-293.

19. McClellan J, Kowatch R, Findling RL; Work Group on Quality Issues. Practice parameter for the assessment and treatment of children and adolescents with bipolar disorder. I Am Acad 
Child Adolesc Psychiatry 2007;46:107-125.

20. Axelson D, Birmaher B, Strober M, Gill MK, Valeri S, Chiappetta L, et al. Phenomenology of children and adoles- cents with bipolar spectrum disorders. Arch Gen Psychiatry 2006;63:1139-1148. 